\title{
TERAPIA DO ESPELHO ASSOCIADA À ESTIMULAÇÃO ELÉTRICA NEUROMUSCULAR PARA REABILITAÇÃO DO MEMBRO SUPERIOR DE PACIENTES HEMIPARÉTICOS POR ACIDENTE VASCULAR CEREBRAL
}

\author{
MIRROR THERAPY ASSOCIATED WITH NEUROMUSCULAR ELECTRICAL \\ STIMULATION FOR UPPER LIMB REHABILITATION IN HEMIPARETIC \\ STROKE PATIENTS
}

\author{
Dionatan KühI \\ Faculdade Guilherme Guimbala \\ Fernando Luís Fischer Eichinger \\ Faculdade Guilherme Guimbala \\ Universidade da Região de Joinville \\ Fabrício Noveletto \\ Faculdade Guilherme Guimbala \\ Universidade do Estado de Santa Catarina
}

\author{
Antonio Vinicius Soares \\ Universidade da Região de Joinville \\ Faculdade IELUSC \\ Contato \\ Antonio Vinicius Soares \\ E-mail: provinicius.soares@gmail.com
}

\section{RESUMO}

Objetivo: Avaliar os efeitos de um programa de reabilitação baseado na combinação da Terapia do Espelho (TE) com a Estimulação Elétrica Neuromuscular (EENM) para o membro superior de pacientes hemiparéticos por Acidente Vascular Cerebral (AVC). Métodos: Trata-se de um estudo pré-experimental envolvendo cinco pacientes com idade média de $53,8( \pm 7,6)$ anos de ambos os sexos. O programa de tratamento consistiu em 20 sessões, com frequência de duas vezes semanais durante 10 semanas. Foram avaliados quanto a força e destreza manual, recuperação motora, espasticidade, independência funcional e percepção da qualidade de vida. Para análise dos dados, foi utilizada estatística descritiva (médias e desvios padrões), e o Teste $t$ de Student para comparar medidas de pré e pós-testes das variáveis controladas no estudo. Resultados: $O$ tratamento baseado na TE associado à EENM trouxe benefícios significativos nas variáveis força e destreza manual, recuperação motora e redução da espasticidade, porém, não houve diferença significativa na percepção de qualidade de vida e independência funcional. Conclusão: Essa combinação parece potencialmente interessante para reabilitação do membro superior de pacientes hemiparéticos por AVC, especialmente para aqueles na fase crônica.

Palavras-chave: Acidente vascular cerebral; Estimulação elétrica; Extremidade superior; Paresia; Modalidades de fisioterapia. 
Objective: To evaluate the effects of a rehabilitation program based on Mirror Therapy (MT) and Neuromuscular Electrical Stimulation (NMES) for the treatment of hemiparetic stroke patients. Methods: This is a pre-experimental study with five patients with average age $53.8( \pm 7.6)$ years, of both sexes. The treatment program consisted of 20 sessions, twice a week for 10 weeks. They were evaluated regarding strength and manual dexterity, motor recovery, spasticity, functional independence, and perception of quality of life. Data analysis was performed by descriptive statistics (means and standard deviations), and Student's $t$ test to compare pre and post-test measures of controlled variables in the study. Results: The treatment has brought benefits to the patients involved in the study on the strength and manual dexterity, motor recovery and reduction of spasticity variables, but it was not able to change the perception of quality of life and functional independence. Conclusion: This combination seems potentially interesting for the rehabilitation of the upper limb of hemiparetic stroke patients, especially for those in the chronic phase.

Keywords: Stroke; Electrical stimulation; Upper extremity; Paresis; Physical therapy modalities.

\section{INTRODUÇÃO}

O Acidente Vascular Cerebral (AVC) é um problema de saúde global comum e incapacitante ${ }^{1}$. O quadro de hemiparesia é observado em $55 \%$ a $75 \%$ dos pacientes e frequentemente ocasiona limitações funcionais no membro superior ${ }^{2}$. A perda funcional no membro superior provoca dificuldades na realização das atividades de vida diária (AVD), tornando o paciente dependente. $O$ alvo na reabilitação do AVC é permitir o maior nível de independência funcional possível para o indivíduo, além do aumento da sua qualidade de vida, levando em consideração todas as limitações existentes ${ }^{3}$.

A neurorreabilitação normalmente é baseada nas técnicas de cinesioterapia convencional, no entanto, esse processo é repetitivo e cansativo para os pacientes ${ }^{4}$. Atualmente, existem diversas técnicas e recursos terapêuticos que são utilizados para a reabilitação do membro superior de indivíduos acometidos pelo AVC, entre elas, estão: treinamento bilateral, terapia de contenção induzida, biofeedback, prática mental, terapia do espelho, treinamento físico, treinamento orientado à tarefa, eletroestimulação, além da inserção de novas técnicas, como a realidade virtual, dispositivos robóticos, neuropróteses, entre outras ${ }^{5-7}$.

A Estimulação Elétrica Neuromuscular (EENM) vem sendo usada por muito tempo na reabilitação de pacientes pós-AVC ${ }^{8}$. Essa técnica gera uma corrente elétrica que, quando aplicada sobre o músculo, induz a contração muscular ${ }^{9}$. Alguns estudos mostraram redução da atrofia muscular, diminuição da espasticidade, aumento da força muscular e facilitação da recuperação do movimento quando utilizada a EENM $^{9-12}$.

Outra abordagem que visa à recuperação funcional do membro superior em pacien- 
tes com AVC é a Terapia do Espelho (TE). É um tratamento relativamente novo para pacientes com AVC, voltado para recuperação da função motora do membro superior por meio da indução de uma reorganização do cérebro ${ }^{13}$. Alguns estudos demonstraram efeitos benéficos da TE na recuperação da função motora vinculada às AVD e na reversão da negligência unilateral ${ }^{14-16}$. A TE utiliza os movimentos do lado não parético do corpo, refletido em um espelho, gerando um feedback visual ${ }^{14,17}$ que permite o treinamento motor bilateral e estimula a recuperação funcional do cérebro ${ }^{18}$. A observação dos movimentos por meio da TE pode ativar neurônios espelhos que estimulam ainda mais o córtex motor afetado $^{19}$. Os neurônios espelhos são um conjunto de células localizadas no córtex pré-motor e no lóbulo parietal inferior, que são estimuladas durante a observação no espelho. Esse sistema de neurônios ajuda a reorganizar o cérebro danificado e a melhorar o controle motor ${ }^{20}$.

Dessa forma, o objetivo deste estudo foi avaliar os efeitos de um programa de reabilitação do membro superior de pacientes hemiparéticos por AVC baseado na TE associada à EENM.

\section{MÉTODOS}

Foi realizado um estudo pré-experimental do tipo pré e pós-teste, envolvendo cinco pacientes com média de idade $53,8( \pm 7,6)$ anos, de ambos os sexos (quatro homens e uma mulher). O tempo médio de lesão foi de 18,8 $( \pm 10,0)$ meses, sendo quatro pacientes que estavam na fase crônica e um na subaguda.
O programa de tratamento teve uma duração de 10 semanas, com frequência de duas sessões semanais, totalizando 20 sessões. O trabalho foi realizado no Núcleo de Pesquisas em Neurorreabilitação do Curso de Fisioterapia da Faculdade Guilherme Guimbala, em Joinville, Santa Catarina. O projeto foi aprovado sob o número de protocolo 051593/2017 pelo Comitê de Ética em Pesquisa em Seres Humanos da Associação Educacional Luterana - Bom Jesus/ IELUSC - Joinville, SC. Todos os participantes assinaram um Termo de Consentimento Livre e Esclarecido.

Foram incluídos na pesquisa pacientes hemiparéticos por AVC clinicamente estáveis, na fase subaguda e crônica. Os critérios de exclusão foram: pacientes que apresentavam hemiparesia decorrente de outras patologias, bem como pacientes hemiplégicos; comprometimento visual e/ou auditivo severo; não cooperativos e/ ou com deficit cognitivo grave; e que tivessem realizado a aplicação da toxina botulínica no membro superior nos últimos seis meses.

Todos os procedimentos de avaliação e tratamento foram realizados pelos próprios pesquisadores.

\section{Instrumentos de medida}

Na triagem inicial, foi utilizado o Miniexame do Estado Mental (MEEM), e os instrumentos específicos foram a dinamometria de preensão manual (Dinamômetro Takei Instruments ${ }^{\circledR}$ ), seguindo as recomendações da Sociedade Americana de Terapeutas da Mão, o Teste de Caixa e Blocos (CB), a Escala de Movimentos 
da Mão (EMM), Escala de Avaliação de FuglMeyer (EFM) - seção referente ao membro superior (pontuação máxima 66 pontos), Escala de Ashworth Modificada (EAM) - (músculos flexores de punho e dedos), Escala de Avaliação da Qualidade de Vida Específica para o AVC (EQV AVC) e o Índice de Barthel Modificado.

\section{Procedimento terapêutico experimental}

A figura 1 ilustra o set experimental utilizado durante as sessões de tratamento com os pacientes. Todos os exercícios com a mão não parética foram realizados na frente do espelho, em que o paciente era orientado a olhar continuamente a imagem refletida no espelho e a realizar as atividades especificas a partir de um feedback auditivo dado pelo fisioterapeuta.
Para o tratamento da TE combinada com a EENM e com atividades manuais especificas, foi utilizado um eletroestimulador $\mathrm{KLD}^{\circledR}$, Modelo ECOR - ET 876, que produz uma corrente bifásica assimétrica com dois canais. O quadro 1 apresenta o protocolo de exercícios utilizados.

\section{Análise de dados}

A tabulação dos dados e a análise estatística foram realizadas no software GraphPad Prism $6^{\circledR}$. Os dados foram analisados por meio de estatística descritiva: média e desvio padrão (para dados paramétricos) e distribuição de frequências com valores absolutos e percentuais (para dados não paramétricos). Para avaliar as diferenças entre as medidas pré e pós-testes de cada variável, foi utilizado o teste $t$ de Student

Figura 1: Set experimental utilizado no tratamento baseado em TE associada à EENM

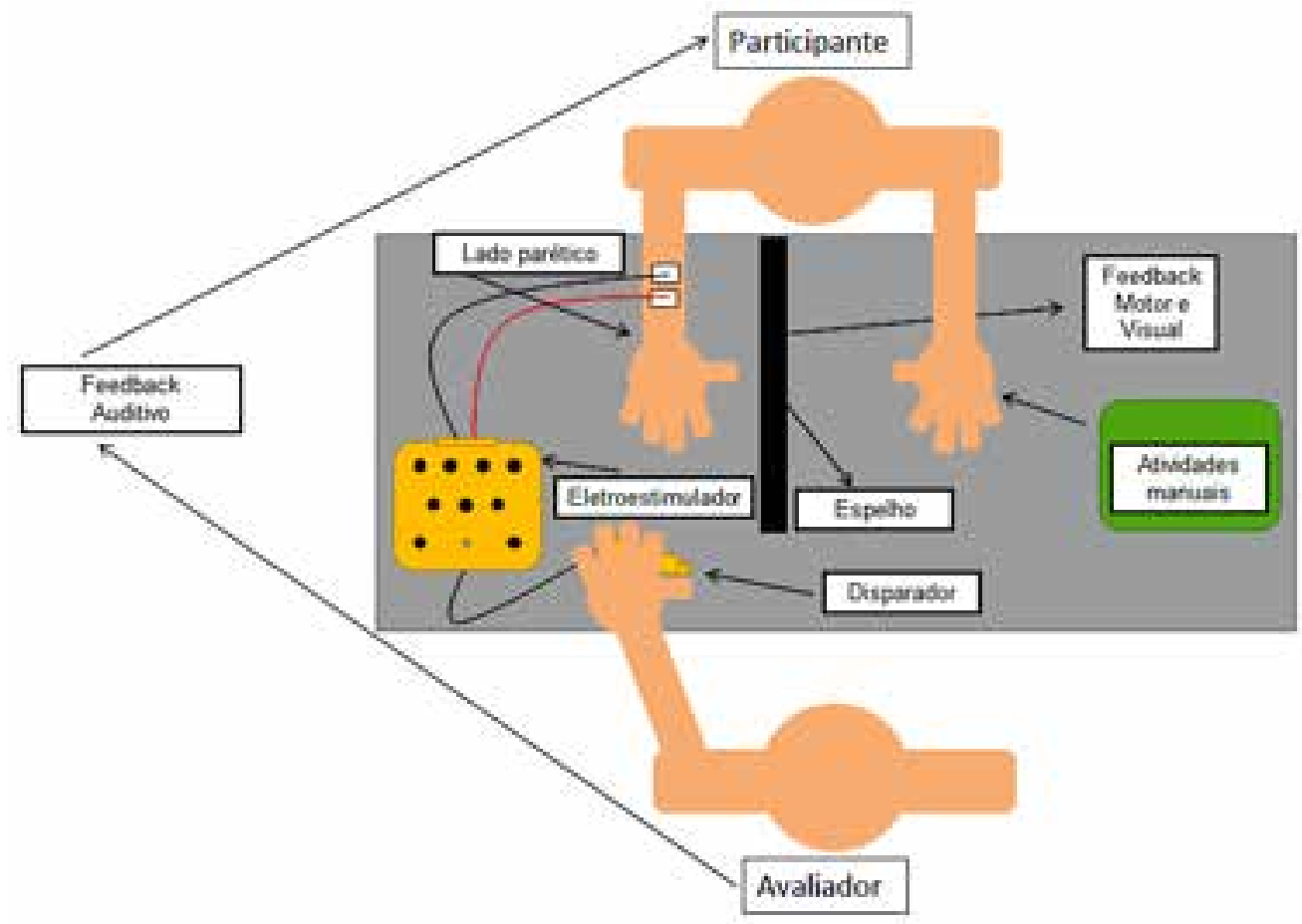

Fonte: Os autores, 2018. 
Quadro 1: Protocolo de exercícios utilizados de forma associada à TE com EENM

\begin{tabular}{|c|l|c|}
\hline Ordem & \multicolumn{1}{|c|}{ Exercício } & Tempo \\
\hline $1^{\circ}$ & $\begin{array}{l}\text { Flexão / Extensão dos dedos } \\
\text { (Aquecimento) }\end{array}$ & 03 minutos \\
\hline $2^{\circ}$ & $\begin{array}{l}\text { Apertar e soltar a bola (Preensão } \\
\text { manual) }\end{array}$ & 03 minutos \\
\hline $3^{\circ}$ & $\begin{array}{l}\text { Apertar e soltar esponja (Preensão } \\
\text { manual) }\end{array}$ & 03 minutos \\
\hline $4^{\circ}$ & $\begin{array}{l}\text { Exercício com Hand Xtrainer } \\
\text { (Extensão resistida dos dedos) }\end{array}$ & 03 minutos \\
\hline $5^{\circ}$ & $\begin{array}{l}\text { Exercício de recolher a faixa } \\
\text { (Flexão dos dedos) }\end{array}$ & 03 minutos \\
\hline $7^{\circ}$ & $\begin{array}{l}\text { Exercício com garrafa (Preensão } \\
\text { manual) }\end{array}$ & 03 minutos \\
\hline $8^{\circ}$ & $\begin{array}{l}\text { Exercício com garrafa (Preensão } \\
\text { manual com prono e supino) }\end{array}$ & 03 minutos \\
\hline $9^{\circ}$ & $\begin{array}{l}\text { Exercício nove buracos e pinos } \\
\text { (Pinçamento) }\end{array}$ & 03 minutos \\
\hline $10^{\circ}$ & $\begin{array}{l}\text { Exercício com a bola em cruz } \\
\text { (Preensão manual e extensão dos } \\
\text { dedos) }\end{array}$ & 03 minutos \\
Cones (Preensão manual e & 03 minutos \\
\hline & \begin{tabular}{l} 
Tempo total de tratamento: 30 minutos \\
\hline
\end{tabular} & \\
\hline
\end{tabular}

Fonte: Os autores, 2018.

Pareado. Foi estabelecido um nível de significância de 95\%. O Tamanho do Efeito (TDE) foi calculado para avaliar os efeitos clínicos da intervenção. Sendo classificado em grande $(\geq 0,8)$, moderado (entre 0,8 e 0,2$)$ e pequeno $(\leq 0,2)$ para os dados paramétricos, e grande $(\geq 0,5)$, moderado (entre 0,5 e 0,1$)$ e pequeno $(\leq 0,1)$ para os dados não paramétricos.

\section{RESULTADOS}

Foram avaliados cinco indivíduos, sendo quatro do sexo masculino e um do sexo feminino, com idade de 53,87,6 anos, que apresentavam quadro de hemiparesia decorrente de AVC e que atendiam aos demais critérios estabelecidos para o estudo. Destaca-se que, durante o experimento, não ocorreram desistências. A tabela 1 apresenta a caracterização sociodemográfica e antropométrica dos participantes, enquanto a tabela 2 apresenta uma caracterização clínica.

No presente estudo, foram incluídos apenas pacientes em fase subaguda e crônica do AVC, devido ao fato de serem observadas melhoras espontâneas nesses pacientes em fase aguda (primeiros três meses pós-AVC) ${ }^{6}$.

Em relação às características clínicas dos pacientes, foi constatado que a maioria dos deles sofreu AVC do tipo isquêmico (60,0\%), e com predomínio do quadro de hemiparesia no dimídio corporal esquerdo $(80,0 \%)$. 
Tabela 1: Características sociodemográficas e antropométricas dos participantes

\begin{tabular}{|c|c|c|}
\hline $\begin{array}{l}\text { Características } \\
\text { Sociodemográficas e } \\
\text { Antropométricas }(n=5)\end{array}$ & & $f(\%)$ \\
\hline \multicolumn{3}{|l|}{ Gênero } \\
\hline Feminino & $\cdots$ & $1(20,0)$ \\
\hline Masculino & --- & $4(80,0)$ \\
\hline Idade (anos completos) & $53,87,6$ & $\cdots$ \\
\hline \multicolumn{3}{|l|}{ Lateralidade autorrelatada } \\
\hline Destro & $\cdots$ & $4(80,0)$ \\
\hline Sinistro/Canhoto & 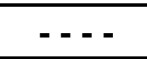 & $0(0)$ \\
\hline Ambidestro & $-\ldots$ & $1(20,0)$ \\
\hline
\end{tabular}

- n: amostra total; f: frequência absoluta; (\%): frequência relativa.

Tabela 2: Características clínicas dos participantes

\begin{tabular}{|c|c|c|}
\hline $\begin{array}{l}\text { Características Clínicas } \\
(n=5)\end{array}$ & & $f(\%)$ \\
\hline \multicolumn{3}{|l|}{ Hemiparesia } \\
\hline Esquerda & - & $4(80,0)$ \\
\hline Direita & $\ldots$ & $1(20,0)$ \\
\hline Tempo de AVC (meses) & $18,810,0$ & \\
\hline \multicolumn{3}{|l|}{ Fase do AVC } \\
\hline Subaguda & $\ldots$ & $1(20,0)$ \\
\hline Crônica & $\bar{n}$ & $4(80,0)$ \\
\hline \multicolumn{3}{|l|}{ Tipo de AVC } \\
\hline Isquêmico & $\ldots$ & $3(60,0)$ \\
\hline Hemorrágico & $\ldots$ & $2(40,0)$ \\
\hline
\end{tabular}

- n: amostra total; f: frequência absoluta; (\%): frequência relativa. 
A tabela 3 apresenta os resultados dos testes de comparação entre as medidas pré e pósintervenção, de todas as variáveis estudadas.

Os resultados dos testes de comparação entre as medidas de pré e pós-testes revelam melhoras estatisticamente significativas com moderado a grande tamanho de efeito em todas as variáveis estudadas, com exceção da percepção de qualidade de vida e da independência funcional, que não foram significativas, embora com grande tamanho de efeito para a variável avaliada com o Índice de Barthel Modificado (IBM). Destaca-se que as principais melhoras estão relacionadas com a espasticidade dos flexores de punho e dedos $(p=0,007$ TDE $=1,7 / p=0,005$ TDE=3,1), controle motor da mão $(p=0,005$ TDE=1,0) e função motora do membro superior $(p=0,006$ TDE=1,1).

Tabela 3: Resumo dos resultados das medidas de pré e pós-testes

\begin{tabular}{|l|l|l|l|l|}
\hline Pacientes (n=5) & Pré () & Pós () & $\mathbf{p}$ & TDE \\
\hline FPM Parético (kgf) & $9,3 \pm 7,2$ & $11,9 \pm 7,5$ & $0,002^{*}$ & 0,4 \\
\hline FPM Não parético (kgf) & $28,1 \pm 8,5$ & $30,0 \pm 8,8$ & $0,002^{*}$ & 0,3 \\
\hline TCB Parético & $13,3 \pm 11,9$ & $16,7 \pm 14,9$ & $0,021^{*}$ & 0,3 \\
\hline TCB Não parético & $36,1 \pm 7,2$ & $44,2 \pm 10,9$ & $0,028^{*}$ & 0,9 \\
\hline EMM & $3,0 \pm 1,5$ & $4,8 \pm 1,6$ & $0,005^{*}$ & 1,0 \\
\hline EFM & $24,3 \pm 17,4$ & $41,6 \pm 13,9$ & $0,006^{*}$ & 1,1 \\
\hline EAM Flexores punho & $3,8 \pm 1,2$ & $1,9 \pm 1,0$ & $0,007^{*}$ & 1,7 \\
\hline EAM Flexores dedos & $4,0 \pm 0,7$ & $1,7 \pm 0,8$ & $0,005^{*}$ & 3,1 \\
\hline EQV AVC & $180,0 \pm 39,6$ & $188 \pm 44,8$ & 0,625 & 0,2 \\
\hline IBM & $72,0 \pm 17,5$ & $83,0 \pm 10,4$ & 0,098 & 0,8 \\
\hline
\end{tabular}

* Indica diferença estatisticamente significativa no teste $t$ de Student Pareado $(p<0,05)$; TDE: Tamanho do efeito; FPM: Força de pressão manual; TCB: Teste de Caixa e Blocos; EMM: Escala de Movimentos da Mão; EFM: Escala de Fugl-Meyer; EAM: Escala de Ashworth Modificada; EQV AVC: Escala de qualidade de vida; IBM: Índice de Barthel Modificado. 


\section{DISCUSSÃO}

Os resultados obtidos a partir da TE associada à EENM trouxeram benefícios para ambos os membros superiores quanto ao controle motor, força de preensão, destreza e redução da espasticidade. Essas alterações, aparentemente, pela percepção dos pacientes, também beneficiaram a independência funcional.

Detalhando os efeitos terapêuticos encontrados neste estudo, pode-se citar o incremento significativo com grande tamanho de efeito sobre a força de preensão manual em ambos os lados, parético $(p=0,002$ TDE $=0,4)$ e não parético $(p=0,002$ TDE $=0,3)$. Os achados obtidos em uma pesquisa realizada por Cho et al. $(2015)^{21}$, que avaliou 27 hemiparéticos por AVC, convergem com o atual resultado: os autores relacionam a TE associada com EENM, que resulta na ativação da região frontoparietal, em que localizam-se populações de neurônios espelho, assim podendo estimular o aprendizado de uma nova habilidade, além de melhorar a sua compreensão.

Com relação à variável destreza manual, foram obtidos ganhos significativos de forma bilateral, com moderado tamanho de efeito no membro parético $(T D E=0,3)$ e grande efeito no membro não parético ( $\mathrm{TDE}=0,9)$; essa melhora explica-se pelo fato de as atividades serem realizadas com o lado não parético. Dois estudos que avaliaram pacientes após a TE associada à EENM observaram a melhora significativa na função motora e na destreza manual ${ }^{21,22}$. Em outro estudo que avaliou a função motora em pacientes pós-AVC e comparou dois grupos diferentes, um com TE associada à FES versus o grupo controle (reabilitação convencional), foi observada melhora superior na destreza manual dos pacientes que realizaram a EENM associada à TE, quando comparado ao grupo controle ${ }^{23}$.

Foram constatadas melhoras estatisticamente significativas e com grande tamanho de efeito $(p=0,005 \mathrm{TDE}=1,0)$ na variável controle motor da mão, avaliada pela EMM, que foi indicada como um bom instrumento de avaliação e predição da recuperação do membro superior parético por $\mathrm{AVC}^{24}$.

A TE associada à EENM e as atividades específicas no presente estudo obtiveram uma melhora significativa na recuperação motora avaliada pela EFM. Em outro trabalho, que também avaliou o efeito da TE associada à EENM, no mesmo público, e utilizando o mesmo instrumento de avaliação, foi obtido o mesmo nível de significância ${ }^{25}$.

$\mathrm{Na}$ avaliação da espasticidade avaliada pela EAM, observou-se uma redução significativa da espasticidade. Esses mesmos resultados foram encontrados por outros pesquisadores, que também utilizaram essa combinação de tratamento ${ }^{26}$. Já em outra pesquisa que utilizou isoladamente a TE, movimentos bilaterais isolados e tarefas funcionais, não se encontrou diferença significativa para espasticidade ${ }^{22}$. Vale lembrar que a associação da EENM pode ter influenciado decisivamente para esse efeito sobre o tônus muscular ${ }^{27}$. 
Embora não tenha sido observada melhora significativa na percepção de qualidade de vida dos pacientes utilizando-se a EQV AVC $(p=0625)$, foi constatado um TDE moderado $(0,2)$, portanto, os resultados encontrados nessa variável não devem ser subestimados. Nessa escala, os escores inferiores a $60 \%$ (<147 pontos) da pontuação total apontam baixa qualidade de vida ${ }^{28}$. $O$ fato de os participantes deste estudo apresentarem escores superiores a 180 mesmo antes do tratamento é uma hipótese para justificar a ausência de incrementos significativos nessa variável. Cabe salientar que a qualidade de vida é uma variável multifatorial, que pode ser influenciada por vários aspectos distintos ${ }^{29}$.

As principais limitações do estudo são relativas ao pequeno número de participantes e à falta de controle de variáveis intervenientes, como o humor, a atenção e o nível de motivação. Tais aspectos são difíceis de controlar, mas sabe-se que podem interferir na adesão e nos desfechos do tratamento. Por mais que tenham sido observados neste estudo preliminar evidentes benefícios aos pacientes, sabemos da necessidade de testar essa combinação de recursos terapêuticos em um número maior de pacientes; e, ainda, verificar se é superior às outras técnicas $e$ métodos utilizados convencionalmente na prática clínica.

\section{CONSIDERAÇÕES FINAIS}

Este estudo preliminar aponta que esta combinação de tratamento envolvendo a TE e a EENM parece ser promissora para recuperação do membro superior de pacientes hemiparéticos por AVC, sobretudo daqueles mais comprometidos e na fase crônica de evolução. Infelizmente, pouco se reserva a esses pacientes pela complexidade do quadro resultante de graves lesões.

A evolução do projeto prevê o recrutamento de um número maior de pacientes e de um grupo controle para verificar se esta proposta de tratamento é superior à intervenção com fisioterapia convencional, uma vez que exige outros materiais e equipamentos para sua implementação. 


\section{REFERÊNCIAS}

1. Faralli A, Bigoni M, Mauro A, Rossi F, Carulli D. Noninvasive strategies to promote functional recovery after stroke. Neural Plast 2013;2013:854597. Epub 2013 Jun 24.

2. Kim H, Shim J. Investigation of the effects of mirror therapy on the upper extremity functions of stroke patients using the manual function test. J Phys Ther Sci 2015;27(1):227-9.

3. Gurbuz N, Afsar SI, Ayas S, Cosar SNS. Effect of mirror therapy on upper extremity motor function in stroke patients: a randomized controlled trial. J Phys Ther Sci 2016;28(9):2501-2506.

4. Soares AV, Woerner SS, Andrade CS, Mesadri TJ, Bruckheimer AD, Hounsell MS. The use of Virtual Reality for upper limb rehabilitation of hemiparetic Stroke patients. Fisioter mov 2014;27(3):309-317.

5. Paz CCSC, Salmela LFT, Criollo CJT. The addition of functional task-oriented mental practice to conventional physical therapy improves motor skills in daily functions after stroke. Phys Ther. 2013;17(6):564-571.

6. Langhorne P, Bernhardt J, Kwakkel G. Stroke rehabilitation. Lancet 2011; 377:1693-702.

7. Iosa MMG, Fusco A, Bragoni M, Coiro P, Multari M. Seven capital devices for the future of stroke rehabilitation. Stroke Res Treat; 2012.

8. Malhotra S, Rosewilliam S, Hermens H. A randomized controlled trial of surface neuromuscular electrical stimulation applied early after acute stroke: effects on wrist pain, spasticity and contractures. Clin Rehabil 2013; (27):579-590.

9. Knutson JS, Fu MJ, Sheffler LR, Chae J. neuromuscular electrical stimulation for motor restoration in hemiplegia. Phys Med Rehabil Clin N Am 2015; 26(4):729-45.

10. Kim JH, Lee LK, Lee JU. A pilot study on the effect of functional electrical stimulation of stroke patients in a sitting position on balance and activities of daily living. J Phys Ther Sci 2013;(25): 1097-1101.

11. Kim MY, Kim JH, Lee JU. The effects of functional electrical stimulation on balance of stroke patients in the standing posture. J Phys Ther Sci 2012;(24):77-81.

12. Levine M, Mcelroy K, Stakich V. Comparing conventional physical therapy rehabilitation with neuromuscular electrical stimulation after TKA. Ortopedia 2013;36.

13. Wu CY, Huang PC, Chen YT, Lin KC, Yang HW. Effects of mirror therapy on motor and sensory recovery in chronic stroke: a randomized controlled trial. Arch Phys Med Rehabil 2013;94:1023-30.

14. Sütbeyaz S, Yavuzer G, Sezer N, Koseoglu BF. Mirror therapy enhances lower-extremity motor recovery and motor functioning after stroke: a randomized controlled trial. Arch Phys Med Rehabil 2007;88:(5):555-9. 
15. Dohle C, Püllen J, Nakaten A, Küst J, Rietz C, Karbe H. Mirror therapy promotes recovery from severe hemiparesis: a randomized controlled trial. Neurorehabil Neural $\operatorname{Re} 2009 ; 23(3): 209-17$.

16. Ji SG, Cha HG, Kim MK, Lee CR. The effect of mirror therapy integrating functional electrical stimulation on the gait of stroke patients. J Phys Ther Sci 2014;26(4):497-499.

17. De Medeiros CSP, Fernandes SGG, Lopes JM, Cacho EWA, Chaco RO. Efeito da terapia de espelho por meio de atividades funcionais e padrões motores na função do membro superior pós-acidente vascular encefálico. Fisioter Pesq 2014;21(3):264-270.

18. Yavuzer G, Selles R, Sezer N. Mirror therapy improves hand function in subacute stroke: a randomized controlled trial. Arch Phys Med Rehabil 2008;89:393-398.

19. Ramachandran VS, Altschuler El. The use of visual feedback, in particular mirror visual feedback, in restoring brain function. Brain 2009;132:1693-710.

20. Carvalho D, Teixeira S, Lucas M, Yuan TF, Chaves F, Peressutti C. The mirror neuron system in post-stroke rehabilitation. Int Arch Med 2013;6:41.

21. Cho HS, Cha HG. Effect of mirror therapy with tDCS on functional recovery of the upper extremity of stroke patients. J Phys Ther Sci 2015;27(4):1045-47.

22. Samuelkamaleshkumar S, Reethajanetsureka S, Pauljebaraj P, Benshamir B, Padankatti SM, David JA. Mirror therapy enhances motor performance in the paretic upper limb after stroke: a pilot randomized controlled trial. Arch Phys Med Rehabil 2014;95(11):2000-5.

23. Kim H, Lee G, Song C. Effect of functional electrical stimulation with mirror therapy on upper extremity motor function in poststroke patients. J Stroke Cerebrovasc Dis 2014;23(4):655-61.

24. Soares AV, Kerscher C, Uhlig L, Domenech SC, Junior NGB. Escala de movimentos da mão: um instrumento preditivo da recuperação funcional do membro superior de pacientes hemiparéticos por acidente vascular cerebral. ACM arq catarin med 2011;40:(2).

25. Yun GJ, Chun MH, Park JY, Kim BR. The synergic effects of mirror therapy and neuromuscular electrical stimulation for hand function in stroke patients. Ann Rehabil Med 2011;35(3):316-21.

26. Lin KC, Chen YT, Huang PC, Wu CY, Huang WL, Yang HW. Effect of mirror therapy combined with somatosensory stimulation on motor recovery and daily function in stroke patients: a pilot study. J Formos Med Assoc 2014;113(7):422- 8.

27. Stein C, Fritsch CG, Robinson C, Sbruzzi G, Plentz RDM. Effects of electrical stimulation in spastic muscles after stroke. Stroke 2015;46(8):2197-205.

28. Rangel ESS, Belasco AGS, Diccini S. Qualidade de vida de pacientes com acidente vascular cerebral em reabilitação. Acta paul. enferm 2013;26(2):205-12.

29. Tastekin N. rehabilitation and quality of life in stroke patients. Turkish J Phys Med and Rehab 2015;61(1):97-8. 